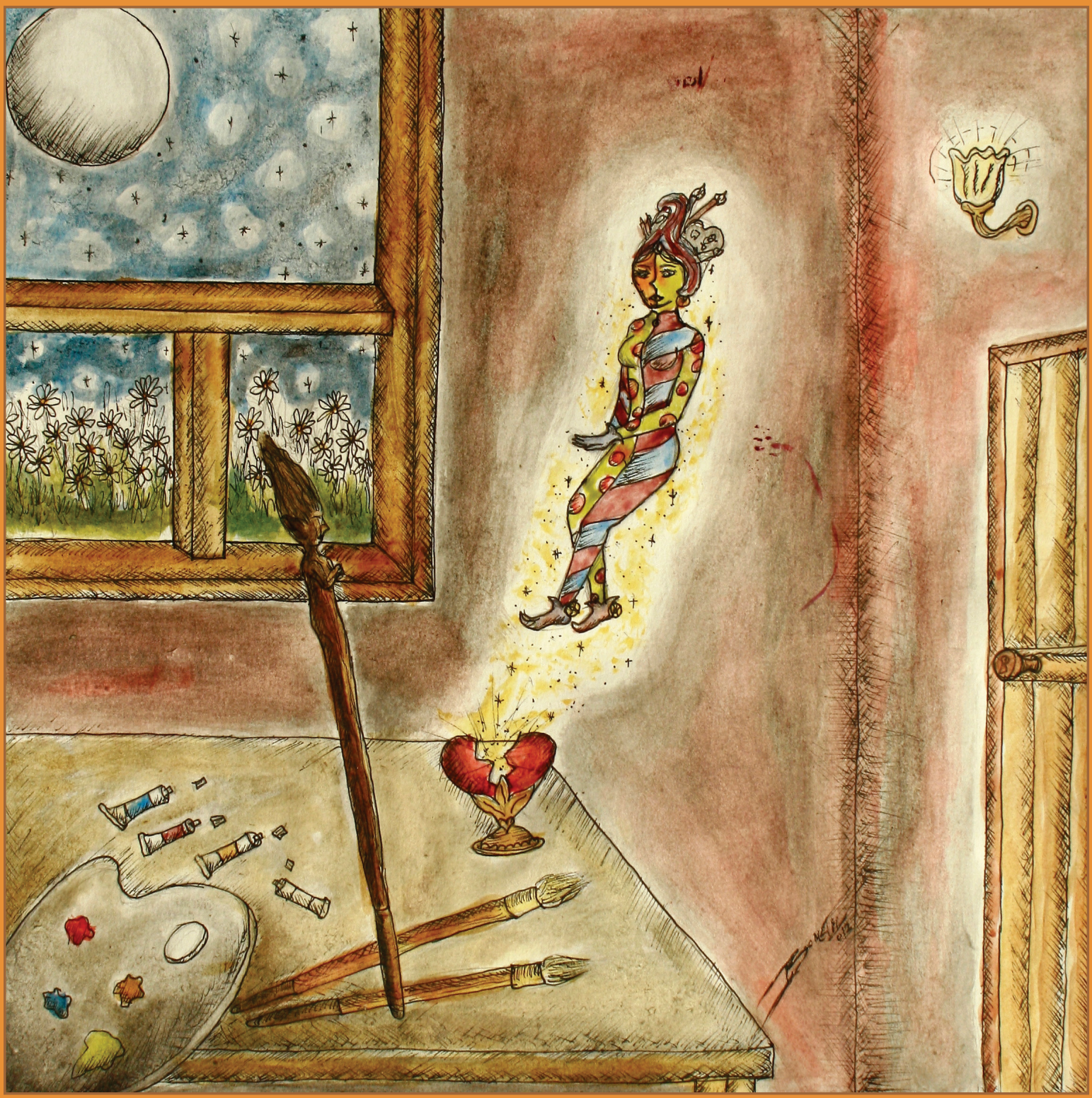

Lorna Boneth Melo - Arte sin Fronteras - Programa Talentos Especiales

Diana Marcela Laverde Robayo

Fonoaudióloga

Especialista en Rehabilitación de la discapacidad de la comunicación infantil

Docente Investigadora - Corporación Universitaria Iberoamericana

diana.laverde@iberoamericana.edu.co 


\title{
REFLEXIONES SOBRE LA UTILIDAD DE LA REALIDAD VIRTUAL EN LA PRÁCTICA FONOAUDIOLÓGICA
}

\section{Considerations about the usefulness of virtual reality at the language -speech therapy practice}

\author{
Fecha de recepción: 30 de julio de 2014 - Fecha de aprobación: 1 de diciembre de 2014
}

\begin{abstract}
RESUMEN
El presente artículo de reflexión refiere la importancia de adherir, de forma frecuente y suficiente, las nuevas Tecnologías de la Información y la Comunicación (TIC) a las prácticas fonoaudiológicas. Enfatiza en la conceptualización y uso de la Realidad Virtual (RV) en tanto se convierte en una herramienta tecnológica que, con seguridad, posibilitará avances importantes en la evaluación y la intervención fonoaudiológica con diferentes poblaciones. Se ubica el concepto de Tecnología de Rehabilitación y, como parte de este, el de Realidad Virtual; la cual se define como un sistema tecnológico, basado en el empleo de computadores y otros dispositivos, cuyo fin es producir una apariencia de realidad que permita al usuario tener la sensación de estar presente en ella. Tiene dos características importantes: la interacción y la inmersión. Se soporta la utilidad de la RV en los procesos de intervención terapéutica desde otras áreas del conocimiento como la Fisioterapia, la Terapia Ocupacional y la Psicología puesto que no existen evidencias de su utilidad en la práctica fonoaudiológica.
\end{abstract}

PALABRAS CLAVE

TIC, Tecnología de Ayuda, Realidad Virtual, Intervención terapéutica con Realidad Virtual.

\section{ABSTRACT}

This document is a consideration about the importance of connecting, frequently and sufficiently, the new Information and Communication Technologies (ICT) with the language-speech therapy practices. It emphasizes in the conceptualization and useful of Virtual Reality (VR) since it is a technological tool that it will make significant advances in the language-speech therapy evaluation and intervention with several populations. It sets the concept of Rehabilitation Technology and, inside this, the concept of VR. The VR is a technological system that is supported by the use of computers and other devices. Its main target is to create produce a visual image of real with the similarities of the real world to the user and it lets his/her has a sensation that is on it. The VR has two principal features: the interaction and the immersion. The useful of VR at the therapeutic intervention in others knowledge areas such as Physical Therapy, Occupational Therapy and Psychology because it does not exist evidences about its practicality in the language-speech therapy practices.

KEY WORDS

ICT, Assistive Technology, Virtual Reality, Therapeutic intervention with Virtual Reality. 
acústica, óptica o electromagnética. Las TIC incluyen la electrónica como tecnología base que soporta el desarrollo de las telecomunicaciones, la informática y el audiovisual.

Particularmente, Sánchez (2003) expone que el contexto en el cual se ha procurado mayor extensión en el conocimiento y uso de las TIC es el educativo. El autor afirma que la introducción de las TIC en el sistema educativo ha permitido un aumento de los recursos didácticos para ser utilizados por los profesores de aula dentro de las diferentes áreas del currículo. Adiciona que es necesario comprender la integración de las TIC en la malla curricular a fin de usar estas tecnologías para lograr un propósito en el aprender de un concepto, un proceso, un contenido, en una disciplina curricular específica. Se trata de valorar las posibilidades didácticas de las TIC en relación con objetivos y fines educativos (Sánchez, 2003).

Para dar cumplimiento al propósito establecido, este artículo se ha organizado en tres apartes. En primer lugar, se exponen las generalidades acerca de las nuevas Tecnologías de la Información y la Comunicación - TIC. En segundo lugar, se presenta información referente a las Tecnologías de Ayuda o de la Rehabilitación. Finalmente, se define y se caracteriza la RV y se sustentan los avances que en esta materia se han obtenido desde otras áreas del conocimiento como la Ingeniería de la rehabilitación, la Psicología, la Terapia Ocupacional y la Fisioterapia. Esta información permite considerar que el diseño e implementación de RV, como Tecnología de ayuda, proporcionará al usuario en condición de discapacidad, el acceso a situaciones y contextos comunicativos reales facilitando el entrenamiento terapéutico, la transferencia de los aprendizajes y los avances significativos y rápidos en los comportamientos comunicativos.

\section{DESARROLLO DEL TEMA}

En forma general, se puede afirmar que las TIC surgen como respuesta a un mundo cambiante, que sigue el ritmo de continuos avances científicos y de la globalización económica y cultural generando la obsolescencia de los conocimientos, el surgimiento de nuevos valores y las transformaciones continuas en las estructuras sociales (Marqués, 2000). Dichas transformaciones han permitido consolidar la sociedad de finales del siglo XX y principios del siglo XXI como la Sociedad del Conocimiento o de la Información, por el papel predominante que ésta ha tomado como bien de consumo (Alcantud y Soto, 2003). Es así como los sistemas informáticos, de telemática y multimedia, los medios de comunicación social mass media y de comunicación interpersonal con soporte tecnológico (teléfono, fax, entre otros) se constituyen hoy día en las denominadas TIC; y permiten que la información sea generada, transmitida, retroalimentada, ampliada, corregida e, incluso distorsionada, a una velocidad inimaginable (Alcantud y Soto, 2003). En consecuencia, el hecho que el uso de las TIC en la vida actual sea tan amplio implica necesariamente que el uso de hardware y software esté cada vez más extendido $y$, por lo tanto, que las personas deban poseer conocimientos teóricos y habilidades prácticas para incluirlos en sus vidas personales, escolares y/o laborales.

Rosario (2006) presenta las TIC como el conjunto de tecnologías que permiten la adquisición, producción, almacenamiento, tratamiento, comunicación, registro y presentación de informaciones, en forma de voz, imágenes y datos contenidos en señales de naturaleza

Lo anterior concuerda con el Plan Decenal de Educación (Ministerio de Educación Nacional de Colombia, 2006) propuesto para Colombia, el cual incluye como uno de los fines, en el Capítulo I: "Desafíos de la Educación, la necesidad de garantizar el acceso, uso y apropiación crítica de las Tecnologías de la Información y la Comunicación como herramientas para el aprendizaje, la creatividad, el avance científico, tecnológico y cultural que permitan el desarrollo humano y la participación activa en la sociedad del conocimiento". Dichas posibilidades de acceso, uso y apropiación son especificadas para la totalidad de la población, incluyendo las personas en condición de discapacidad.

Por tal motivo, los profesionales de la rehabilitación, entre los cuales se ubican los fonoaudiólogos, deben también procurar el acceso y uso de las TIC por parte de la población con deficiencias para la comunicación y/o discapacidad comunicativa. Esta tarea inaplazable requiere que los fonoaudiólogos conozcan, diseñen y utilicen de forma apropiada y oportuna las herramientas tecnológicas.

En tal sentido, conviene citar a Cuervo (1999a), quien enfatiza que la función terapéutica o intervención fonoaudiológica involucra, entre otras, acciones como: utilizar tecnología computarizada o de otra índole para el registro y la medición de las dimensiones comunicativas, la retroalimentación y el monitoreo de respuestas, y el uso de sistemas de comunicación aumentativa y/o alternativa. Igualmente, la autora recomienda que la estructura de los servicios fonoaudiológicos, debe constituirse por componentes como planta física, equipos, materiales y personal idóneo, para garantizar la calidad de los mismos. Cada uno de estos componentes es susceptible de inspección y verificación e informan sobre la capacidad del programa fonoaudiológico para ofrecer atención (Cuervo, 1999b). Con respecto específicamente al componente de equipos y materiales, Cuervo (1999b) señala que "frente al avance del conocimiento sobre la naturaleza, el diagnóstico y el tratamiento de los desórdenes de comunicación y la consecuente construcción de tecnología en estas áreas, la cantidad y la calidad de los recursos a disposición de un fonoaudiólogo determinan hoy la calidad de los servicios que ofrece" (p. 164).

En respuesta a estos lineamientos, para el abordaje de las deficiencias y la discapacidad, se asumen las TIC como herramientas fundamentadas en la informática y con costo mínimo, que tienen 
como finalidad compensar o sustituir aquellas funciones que se encuentran disminuidas o alteradas. Estas herramientas permiten a las personas que las requieren su aprovechamiento máximo con bajo desgaste de energía (mecánica o física) e independencia de las personas de su entorno (Sánchez, 2002). Mediante la implementación de las TIC, los usuarios en situación de discapacidad pueden acceder tanto a contenidos académicos como al manejo y aprendizaje de destrezas laborales, mediante la adaptación de hardware y software (Sánchez, 2002).

Diversos autores como Cook, Hussey, Bain (citados por Fuentes y Sotomayor, 2009) han referido las TIC, en el campo de la discapacidad, con diferentes denominaciones, a saber: Tecnología de la Rehabilitación, Tecnología de Ayuda, Tecnología de Asistencia, Tecnología de Adaptación y Tecnología de Acceso. Sin embargo, pese a la variedad de denominaciones, estas tecnologías pueden definirse como "cualquier elemento, pieza, dispositivo, sistema o servicio que es utilizado para incrementar, mantener o mejorar las capacidades funcionales de personas con deficiencias y/ con discapacidad" (The Technology-Related Assistance for Persons with Disabilities Act, 1988, citado por Pena et.al. 1999, p. 1).

A su vez, Alcantud y Soto (2003) consideran que las Tecnologías de Ayuda son un método de trabajo que persigue compensar o potenciar las facultades sensoriales, físicas o mentales de las personas en condición de discapacidad. Dentro de este contexto se pueden encontrar recursos tecnológicos orientados específicamente a la rehabilitación de discapacidades comunicativas y/o a la comunicación alternativa/aumentativa.

Según Fuentes y Sotomayor (2009), el desarrollo y los productos de Tecnología de Rehabilitación comprenden 2 tipos de actuación: a) Diseño Público y b) Ayudas Técnicas. El Diseño Público hace referencia a productos y servicios dirigidos al público en general, e incorporan, en la medida de lo posible, un diseño que sea accesible para el mayor número de personas de acuerdo con el grado de sus habilidades. Por otro lado, las Ayudas Técnicas son productos y servicios dirigidos a solucionar un problema de accesibilidad generado por la existencia de discapacidad, que no puede ser solventado por productos de uso corriente. El diseño de Ayudas Técnicas se realiza teniendo en cuenta las condiciones funcionales de acuerdo con las habilidades físicas, sensoriales y psíquicas de las personas.

Así mismo, en el marco de las Tecnologías de la Rehabilitación o de Ayuda se pueden contemplar los sistemas informáticos entre los cuales se ubican las Realidades Virtuales. Una Realidad Virtual (RV) se define como un sistema tecnológico, basado en el empleo de computadores y otros dispositivos, cuyo fin es producir una apariencia de realidad que permita al usuario tener la sensación de estar presente en ella (Burdea y Coiffet, citados por Wang y Reid, 2013; Yagamuchi, Foloppe, Richard, Richard, y Allain, 2012).

Según Gálvez (2004) la virtualidad permite una nueva forma de relación entre el uso de las coordenadas de espacio y de tiempo, supera las barreras espacio-temporales y configura un entorno en el que la información y la comunicación se muestran accesibles desde perspectivas hasta ahora desconocidas, al menos en cuanto a sus volúmenes y posibilidades. La RV permite la generación de entornos de interacción que separan la necesidad de compartir el espacio-tiempo, facilitando en este caso nuevos contextos de intercambio y comunicación. En tal sentido, la RV ha eliminado la frontera existente entre realidad e irrealidad.

Igualmente, Gálvez (2004) afirma que la amplia variedad de posibilidades que ofrece la RV, ha facilitado el establecimiento de un estatus de realidad, sustentado fundamentalmente en tres aspectos: a) La RV es compartida con otras personas. Se centra generalmente en la interacción interpersonal, que a pesar de no producirse en el mismo espacio-tiempo, si es percibida como un acto colectivo; b) Tiene una estrecha relación con el mundo físico dada su interrelación e influencia mutua. La experiencia en la realidad virtual viene mediada por la experiencia en el mundo real y ésta es influida por lo que allí es experimentado; y c) Está interconectada con la producción artística, ya que se convierte en un espacio de creación con motivaciones estéticas.

En resumen, las aplicaciones en RV tienen dos características principales: la interacción y la inmersión. La interacción computador-ser humano sucede a través de múltiples canales sensoriales que llevan a las personas a explorar los ambientes virtuales mediante la vista, la audición y el tacto (Burdea y Coiffet, 1994, citados por Wang y Reid, 2013). Por su parte, la inmersión se refiere al grado en el cual la persona se siente involucrada o envuelta en el ambiente virtual (Wang y Reid, 2013), además, la inmersión tiene que ver con los tipos de hardware y software utilizados. En tal sentido, los sistemas de RV se clasifican en inmersivos y no inmersivos.

Los sistemas inmersivos son aquellos ligados a un entorno virtual generado por un computador, en el cual el sujeto para interactuar lo hace a través de algún tipo de hardware, como guantes, cascos, pantallas, cabinas o cuevas virtuales. En cambio, en los sistemas no inmersivos la interacción con el entorno virtual se realiza mediante un teclado, mando o ratón sin que se precise de otro tipo de hardware o periférico adicional conectado al ordenador (Bayón y Martínez, 2009). Igualmente, Bayón y Martínez (2009) reportan que entre los sistemas mayormente utilizados para la rehabilitación física se encuentran: a) Sistemas Inmersivos como: System Interactive Rehabilitation Exercise, Rutgers Master II-ND, Rutgers Ankle Rehabilitation System, CyberGlove; y b) Sistemas no inmersivos que se soportan en las consolas comerciales de bajo coste como Nintendo Wii y Sony Playstation 2, Eye Toy. Estos sistemas proporcionan ejercicios programados para que los usuarios los ejecuten favoreciendo el equilibrio, la marcha, los movimientos del miembro superior y la heminegligencia unilateral.

Dadas las características de este tipo de tecnología y como parte importante de la denominada Tecnología de la Rehabilitación, es requerido que el profesional en Fonoaudiología pudiera utilizarla como parte de su ejercicio de intervención terapéutica con población con deficiencias para la comunicación y/o con discapacidad comunicativa. Sin embargo, debe tenerse en cuenta que el diseño y la implementación de sistemas de RV requieren, por supuesto, un trabajo interdisciplinario cuyo equipo profesional estaría conformado por Ingenieros Biomédicos y de la Rehabilitación, Neurocientíficos, 
Científicos de la computación, y otros profesionales de la Rehabilitación. Las herramientas que estos profesionales desarrollan, satisfacen las necesidades en procesos motores, comunicativos, visuales y cognitivos de las personas y las ayudan a responder a las demandas y tareas de la vida cotidiana relacionadas con la vida independiente, la educación y el trabajo (National Institute of Biomedical Imaging and Bioengineering, 2013).

Autores como Keshner, Weiss, Rand, Katz y Kizony (2004, 2007, citados por Weiss, Rand, Katz y Kizony, 2004) han sustentado la importancia del uso de la RV en rehabilitación. De acuerdo con sus investigaciones, la intervención con $\mathrm{RV}$, específicamente en el área de la rehabilitación física y cognitiva, puede producir un impacto clínico significativo. Además, Rizzo, Schulteis, Kerns, Matter y Kim (1998, 2001, citados por Weiss, Rand, Katz y Kizony, 2004) informan que la RV tiene diversidad de ventajas para la rehabilitación como la oportunidad de aprender activamente a partir de la experiencia; la habilidad para medir el comportamiento de manera objetiva en ambientes seguros y ecológicamente válidos mientras se mantiene el control sobre la presentación de los estímulos; permite individualizar las necesidades de tratamiento mientras que se incrementa la complejidad de las tareas y se elimina progresivamente el soporte de los terapeutas.

A este respecto de la Rehabilitación Neurológica, varios estudios, entre los que se incluyen los mencionados a continuación, muestran evidencias importantes sobre la utilidad de la RV y sus aportes a la neuroplasticidad.

Johnson, Rose, Rushton, Pentland, y Attree (1998) afirman que las capacidades de la RV han sido especialmente notorias en la Rehabilitación Neurológica, ya que posterior a una lesión o daño neurológico, se ubican deficiencias físicas concomitantes con deficiencias cognitivas y comunicativas que reducen los niveles de funcionalidad e interacción de las personas. Los autores consideran que la RV ofrece un potencial considerable para alcanzar éxitos significativos en evaluación, tratamiento y resultados esperados, incrementando el conocimiento en neuroplasticidad.

Así mismo, al reconocer que en la fase aguda posterior a la presentación de un daño cerebral se genera deterioro funcional y neural debido sencillamente a la falta de estimulación cortical, y que muchos procesos terapéuticos son poco prácticos y se prestan en tiempos inapropiados; se ha demostrado que la versatilidad de la RV entra en juego, facilitando la readquisición de habilidades dada la concurrencia de tres factores: el entrenamiento masivo, el tiempo y los ambientes enriquecidos, que permiten la reorganización de los sistemas neuronales. Dicha versatilidad obedece a la presentación de estímulos sensoriales complejos, a través de retroalimentaciones visuales, auditivas y somatosensoriales (Adamovich, Fluet, Tunik, y Merians, 2010).

Adicionalmente, McComasy colaboradores (2003), citados por Sveistrup (2004), consideran que la RV ofrece la capacidad para individualizar los tratamientos necesarios mientras que proporcionan estandarización de protocolos de entrenamiento y tratamiento. De hecho, la evidencia preliminar indica que la RV proporciona un me- dio único donde la terapia se realiza en un contexto intencionado, motivante, funcional y puede ser graduado y documentado.

En los procesos terapéuticos es mayor la cantidad de información disponible para la aplicación de la RV especialmente en las áreas de Rehabilitación Física, Ocupacional y Cognitiva. Esta última implementada especialmente por el área de la Psicología. Sveistrup (2004) reportó que se han encontrado muchos hallazgos sobre la utilidad de la RV en la rehabilitación del movimiento en ambientes virtuales teniendo en cuenta criterios como el desplazamiento, el control postural, la prensión y la fuerza ejercidas para ejecutar un movimiento en relación con los objetos del ambiente virtual. Reporta particularmente la posibilidad de modular las tareas desde una menor complejidad a una mayor complejidad; así como, la transferencia a los ambientes cotidianos reales.

Así mismo, en las áreas de Terapia Física y Ocupacional, Bayón y Martínez (2009) realizaron una recopilación bibliográfica acerca de la utilidad de la RV en la rehabilitación motora de pacientes quienes presentaban ictus embólico. Los hallazgos de su estudio evidenciaron que existen terapias que utilizan la RV con pacientes con daño neurológico, inicialmente en fase experimental, y posteriormente como coadyudante en los procesos de tratamientos convencionales. Hasta el momento se han implementado los programas de RV con usuarios quienes presentan hemiparesia de grado leve con poco deterioro cognitivo. Los autores afirman, que aunque parece que facilitan los fenómenos de reorganización cortical y neuroplasticidad, su utilización August et.al., 2006continúa siendo limitada. Sin embargo, ya han encontrado evidencia de activación de áreas motoras primarias y secundarias mientras se ejercitan la mano o el brazo. También se atribuye la mejoría funcional obtenida, a la reorganización cortical del hemisferio lesionado junto con la disminución de la activación aberrante del córtex contralateral (Jang et.al., 2005). Así mismo, se ha demostrado que el feedback sensorial asociado a estos ejercicios en entorno virtual activaría los sistemas de neuronas en espejo que facilitarían el aprendizaje (Iacoboni et.al. 1999).

Arias, Robles-García, Sanmartín, Flores, y Cudeiro (2012) han realizado estudios importantes sobre la disposición de herramientas en RV para la evaluación e intervención terapéutica en el área de Fisioterapia. Particularmente, su estudio estuvo dirigido a evaluar los movimientos rítmicos de la mano en tres grupos: pacientes con enfermedad de Parkinson, pacientes en proceso de envejecimiento típico y jóvenes saludables, como grupo control. Compararon la ejecución de estos movimientos tanto en el mundo real como en RV. Encontraron que la evaluación en RV es tan válida como en el mundo real ya que no se distorsionan los movimientos y se detectan las alteraciones en la formación rítmica. Este estudio soporta la RV como una herramienta prometedora en el hallazgo de alteraciones en el movimiento rítmico y seguramente en el proceso de tratamiento en diferentes grupos poblacionales.

Con respecto a la Rehabilitación Cognitiva con apoyo en RV también se encuentran investigaciones pertinentes. Butler (2007, citado por Wang y Reid, 2013) afirma que la Rehabilitación Cognitiva tradicional emplea ejercicios específicos, diseñados para mejorar las funciones cognitivas deterioradas, a través de entrenamiento 
repetitivo de ejercicios. Este tipo de ejercitación es necesaria para reorganizar el cerebro en áreas particulares, sin embargo, es altamente demandante tanto para el usuario como para el instructor. Estas demandas representan un gran problema cuando se habla de rehabilitación cognitiva en niños con autismo, ya que ellos son particularmente difíciles de atraer. Entonces, la RV es de gran utilidad puesto que permite que ellos mantengan la atención proporcionándoles actividades estructuradas e individualizadas y direccionan sus debilidades mientran construyen sus fortalezas (Rizzo, Schultheis, Kerns, y Mateer, 2004).

Wang y Reid (2013) han descrito la Rehabilitación Cognitiva con RV en el proceso terapéutico de niños con autismo. Ellos han referido que no existen estudios previos que den cuenta de este tipo de rehabilitación, aunque sí han encontrado investigación que muestra el uso de la RV, con niños y adolescentes con autismo, en tareas específicas como seguimiento de instrucciones, cruzar calles, pedir café y abandonar un edificio que se incendia (Rose et. al., 2000; Strickland, Marcus, Mesibov y Hogan, 1996; Mitchell, Parsons y Leornard, 2007; Self, Scudder, Weheba y Crumrine, 2007).

En su estudio piloto, Wang y Reid (2013) tuvieron como propósito demostrar la utilidad de la Rehabilitación Cognitiva con RV en el mejoramiento de las capacidades de procesamiento contextual de los objetos (abstracción y flexibilidad mental) en 4 niños con autismo; y explorar las percepciones de los padres frente a los cambios comportamentales de sus hijos durante el proceso de tratamiento. Ellos encontraron que los niños lograron avances significativos crecientes durante las 2 semanas de intervención terapéutica, en el procesamiento contextual de los objetos y flexibilidad cognitiva. Sin embargo, no hubo avances significativos en atención sostenida. Igualmente, los padres de 2 de los 4 niños informaron que posterior al entrenamiento con RV, sus hijos podían iniciar una conversación y responder a preguntas más apropiadas durante la misma. Dados los hallazgos, los autores concluyeron que su estudio piloto demuestra la eficacia que tiene la implementación de la RV para Rehabilitación Cognitiva en niños con autismo, pero sugieren hacer estudios a mayor escala para evaluar la magnitud de los cambios resultantes a fin de lograr avances tanto en las actividades de la vida diaria como en la calidad de vida de esta población.

Otro ejemplo del uso de la RV con relación a servicios de cuidado de salud es tomado del estudio de Metin et al (2013). Ellos investigaron la utilidad de la RV en población con parálisis cerebral en el proceso terapéutico de Adaptación Psicológica, teniendo como instrumento de registro de los avances la Escala Hacettepe de Adaptación Psicológica. Los investigadores encontraron diferencias significativas entre el grupo de estudio y el grupo control en cuanto a los resultados de la Escala, antes y después del tratamiento. Además, evidenciaron que la terapia de RV en combinación con las técnicas terapéuticas tradicionales trae mayor éxito en el tratamiento.

Si bien en el campo de la intervención fonoaudiológica se identifican herramientas tecnológicas que son asumidas como Tecnología de Ayuda o Asistente y que son utilizadas por los profesionales en Fonoaudiología durante los diferentes momentos del proceso terapéutico, no se han desarrollado e implementado sistemas de RV. Las
Tecnologías de Ayuda se han ubicado en el espectro de los Sistemas de Comunicación Aumentativa y Alternativa; y se concentran en el uso del computador y de software diversos entre los que pueden citarse la Colección de PIPO, el CyT-L que se constituye en una adaptación en soporte informático del programa de enriquecimiento cognitivo 'Comprender y Transformar', el Software de Habilidades Cognitivas (Innovaciones Software y servicios), las herramientas del Visi-Pitch, Aprendilandia (Planeta de Agostini), entre otros (Moreno y Aguilera, 2002).

\section{CONCLUSIÓN}

Aunque los estudios presentados no son realizados por fonoaudiólogos, ni en ámbitos nacionales ni internacionales, proporcionan un sustento pertinente y suficiente frente a la importancia de iniciar el diseño e implementación de la RV como herramienta que garantice a los usuarios con deficiencias para la comunicación y/o discapacidad comunicativa el acceso a este tipo de tecnología, posibilitando la efectividad y avances realmente significativos en su función interactiva.

En tal sentido y a manera de conclusión, podría decirse que es imperativa la utilización de RV en los procesos terapéuticos realizados por fonoaudiólogos en tanto: a) posibilita las múltiples interacciones y comunicaciones rompiendo, precisamente, con las barreras o limitaciones que imponen el tiempo y el espacio; b) la anterior característica, permite involucrar a los usuarios en situaciones y contextos reales de comunicación que conlleven a la transferencia de las múltiples estrategias y acciones propias de la consulta, en mundo real, y que frecuentemente no logran trascender las paredes del consultorio para convertirse en herramientas útiles durante las interacciones comunicativas; c) puede acompañar el trabajo terapéutico tradicional, no chocan el uno con el otro; d) pueden elaborarse ambientes virtuales utilizando consolas de uso común como el Nintendo Wii y el PlayStation, lo que conllevaría a un bajo costo económico; e) cualifica el ejercicio profesional respondiendo a las exigencias públicas y particulares de la profesión, especificadas en la normativa, los manuales de procedimientos, los programas y proyectos,; f) posibilita el encuentro con otros profesionales para la creación e implementación de la tecnología en RV ampliando los campos de trabajo interdisciplinario en pro de suplir las necesidades de personas en condición de discapacidad comunicativa; g) abre las puertas a la investigación, tanto teórica como aplicada, en áreas hasta el momento inexploradas por los fonoaudiólogos, por lo menos en Colombia, con opciones para la producción científica escrita.

Con base en la información presentada y un conjunto de conclusiones apenas esbozadas, este artículo ha sustentado la necesidad de diseñar e implementar los sistemas de RV en la práctica profesional fonoaudiológica. La invitación es para todos: se requiere indagar, construir, evaluar, conversar y otras tareas más, que permitan dar los primeros pasos para responder a ésta demanda, que no es nueva, pero que sí es coherente con las necesidades de poblaciones con deficiencias para la comunicación y/o discapacidad comunicativa y un mundo en cambio constante. No puede desconocerse que pueden aparecer condiciones limitantes pero así mismo podrán aparecer aquellas condiciones facilitadoras que en definitiva soportarán el proceso. ¿Cuál es su apuesta? 


\section{REFERENCIAS}

Adamovich, S., Fluet, G., Tunik, E., \& Merians, S. (2010). Sensoriomotor training in virtual reality: a review. Neurorehabilitation, 1-21.

Alcantud, F., \& Soto, F. (2003). Tecnología de ayuda en personas con trastornos de comunicación. NAU Llibres.

Arias, P., Robles-García, V., Sanmartín, G., Flores, J., \& Cudeiro, J. (2012). Virtual reality as a tool for evaluation of repetitive rhytmic movements in the elderly and Parkinson's disease patients. Plos One Open Access freely avaliable online.

August, K., Lewis, J., Chandar, G., Merians, A., Biswal, B., \& Adamovich, S. (2006). FMRI analysis of neural mechanisms underlying rehabilitation in virtual reality: activating secondary motor areas. Conf Proc IEEE Eng Med Biol Soc, 3692.

Bayón, M., \& Martínez, J. (2009). Rehabilitación del Ictus mediante realidad virtual. Rehabilitación, 256-260.

Cuervo, C. (1999a). Naturaleza del servicio fonoaudiológico. En C. Cuervo, La profesión de fonoaudiología: Colombia en perspectiva internacional. Pp.127. Bogotá: Universidad Nacional de Colombia.

Cuervo, C. (1999b). Calidad de los servicios fonoaudiológicos. En C. Cuervo, La profesión de fonouadiología: Colombia en perspectiva internacional. Pp. 162-177. Bogotá: Universidad Nacional de Colombia.

Fuentes, Y. \& Sotomayor, E. (2009). Las Tecnologías de la Información y la Comunicación (TIC) como instrumento de ejercicio de derechos. Tabula Rasa Revista de Humanidades, 359-373.

Gálvez, A. (2004). Posicionamientos y puestas en pantalla. Un análisis de la producción de sociabilidad en los entornos virtuales. (Tesis doctoral). Obtenido de Universidad de Barcelona: http://hdl.handle. net/10803/5434

Iacoboni, M., Woods, R., Brass, M., Bekkering, H., Mazziotta, J., \& Rizzolatti, G. (1999). Cortical mechanism of human imitations. Science, 2526-2528.

Jang, S., You, S., Hallet, M., Cho, Y., Park, C., \& Cho, S. (2005). Cortical reorrganization and associated functional motor recovery after virtual reality in patients with chronic stroke: an experimenter-blind preliminary study. Arch Phys Med Rehabil, 2218-2223.

Johnson, D., Rose, F., Rushton, S., Pentland, B. y Attree, E. (1998). Virtual reality: a new prosthesis for brain injury rehabilitation. Scottish $\mathrm{Me}-$ dical Journal.

Marqués, P. (2000). Las TIC y sus aportaciones a la sociedad. Departamento de Pedagogía Aplicada. Facultad de Educación. UAB. Obtenido de http://dewey.uab.es/pmarques/tic.htm
Metin, B., Dogan, M.; Cuhadaroglu, F., Nakipoglu, G., Köse, B. \& Özgirgin, N. (2013). The effect of Virtual Reality Therapy on psychological adaptation in children with cerebral palsy. Archives of Neuropsychiatry. Vol 50. Pp.70-74

Ministerio de Educación Nacional de Colombia. (2006). Plan Decenal de Educación 2006 - 2016. Bogotá.

Mitchell, P., Parsons, S., \& Leornard, A. (2007). Using virtual environments for teaching social understanding to 6 adolescents with autistic spectrum disorders. Journal of Autism and Developmental Disorders., 589-600.

Moreno, F. y Aguilera, A. (2002). Rehabilitación cognitiva en sujetos discapacitados mediante el empleo de nuevas tecnologías: El programa C y T-L. Obtenido de http://ardilladigital.com/DOCUMENTOS/TECNOLOGIA\%20EDUCATIVA/TICs/T6\%20TICS\%20Y\%20NEE/c80.pdf

National Institute of Biomedical Imaging and Bioengineering. (Junio de 2013). Rehabilitation Engineering. Obtenido de www.nibib.nih.gov.

Pena, J., Vásquez, C., Pérez, I., Rodríguez, I., López, J., Planell, D., \& Millanes, J. (1999). Contribuciones de la electrónica y la fotónica a la tecnología de la rehabilitación. Mundo Electrónico.

Rizzo, A., Schultheis, M., Kerns, K., \& Mateer, C. (2004). Analysis of assets form virtual reality applications in neuropsychology. Neurosychological Rehabilitation, 207-239.

Rosario, J. (2006). Observatorio para la Cibersociedad. Obtenido de http:// www.cibersociedad.net/archivo/articulo.php?art=221

Rose, F., Attree, E., Brooks, B., Parslow, D., Penn, P., \& Ambihaipahan, N. (2000). Training in virtual enviroments: transfer to real world tasks and equivalence to real task training. Ergonomics, 494-511.

Sánchez, J. (2003). Integración Curricular de TICs: concepto y modelos. Enfoques Educacionales, 51-65.

Sánchez, R. (2002). Ordenador y Discapacidad: Guía práctica de apoyo para las personas con necesidades educativas especiales. CEPE.

Self, T., Scudder, R., Weheba, G., \& Crumrine, D. (2007). A virtual approach to teaching safety skills to children with autism spectrum disorder. Topics in Language Disorders., 242-253.

Strickland, D., Marcus, L., Mesibov, G., \& Hogan, K. (1996). Brief report: two case studies using virtual reality as a learning tool form autistic children. Journal of Autism and Developmental Disorders, 651-659.

Sveirtrup, H. (2004). Motor rehabilitation using reality virtual. Journal of Neuroengineering and Rehabilitation, 1-10. 
McComas, J., Sveistrup, H., Thornton, M., Finestones, H., McCormick, A., McLean, J., Bryanton, C. (2004). Outcomes of intervention programs using flat screen virtual reality. Proceedings of the 26th Annual International Conferencee of IEEE/EMBS., 4856-4858.

Sveistrup, H., McComas, J., Thornton, M., Marshall, S., Finestones, H., McCormick, A., Mayhew, A. (2003). Experimental studies of virtual reality-delivered compared to convetional exercise programas for rehabilitation. Cyberpsycholgy Behavior, 243-249.

Wang, M., \& Reid, D. (2013). Using the Virtual Reality - Cognitive Rehabilitation approach to improve contextual processing in children with autism. Obtenido de Hindawi Publishing Corporation: http://dx.doi. $\operatorname{org} / 10.1155 / 2013 / 716890$

Weiss, P., Rand, D., Katz, N., \& Kizony, R. (2004, 1:12). Video capture virtual reality as a flexible and effective rehabilitation. Journal of NeuroEngineering and Rehabilitation.

Yagamuchi, T., Foloppe, D., Richard, P., Richard, E., \& Allain, P. (2012). A dual-modal Virtual Reality Kitchen for (re)learning of everyday cooking activities in Alzheimer's Disease. Presence, Vol.21, No.1, Pp.44. 